Thomas Zauner*

\title{
Maria Theresia sagt: Danke!
}

\author{
Die Österreichische Nationalbibliothek konnte ihre erste Crowdfunding-Aktion erfolgreich \\ umsetzen
}

DOI 10.1515/bfp-2017-0028

Zusammenfassung: Die Österreichische Nationalbibliothek startete als erste Nationalbibliothek im deutschsprachigen Raum eine Crowdfunding-Aktion. Von 20. Oktober bis 22. November 2016 konnten Interessierte auf www. wemakeit.com zur Restaurierung eines wertvollen Buches aus der Zeit Maria Theresias beitragen. Schon nach drei Wochen war das Finanzierungsziel von 15500 Euro erreicht, bis zum Ende der Aktion haben 166 Personen insgesamt 26315 Euro gespendet.

Schlüsselwörter: Österreichische Nationalbibliothek; Crowdfunding; Buchrestaurierung

\section{Maria Theresa Says: Thank you! Austrian National Library was Able to Successfully Implement its First Crowdfun- ding Campaign}

\begin{abstract}
The Austrian National Library was the first national library in the German-speaking world to launch a crowdfunding campaign. From 20 October till 22 November 2016, interested persons could contribute to the restoration of a valuable book from the time of Maria Theresa on www.wemakeit.com. 166 donors had paid a total amount of EUR 26,315 until the end of the campaign.
\end{abstract}

Keywords: Austrian National Library; crowdfunding; book conservation

1740 stand zum ersten und einzigen Mal eine Frau an der Spitze des riesigen Habsburgerreiches: Maria Theresia. 23 Jahre jung war die Erzherzogin, als sie die Regierungsgeschäfte von ihrem Vater Kaiser Karl VI. übernahm, der ohne männlichen Erben kurz davor verstorbenen war. Die protokollarisch vorgesehene „Erbhuldigung“ fand am 22. November statt: Dabei bezeugten die Niederösterreichischen Stände der neuen Landesherrin in einem großen Festakt ihre Loyalität. Der Festakt wurde in einem kunst-

*Kontaktperson: Thomas Zauner, thomas.zauner@onb.ac.at voll ausgestatteten Druckwerk festgehalten, das bald als Geschenk an die damalige Hofbibliothek kam. Dort wurde es 200 Jahre lang nicht irgendwo, sondern im Büro des Präfekten gelagert und auch immer wieder den zahlreichen Ehrengästen gezeigt. Leider erfolgten Lagerung und Präsentation - aus heutiger Sicht - unsachgemäß und entsprechend bedauernswert war der Zustand des Werks: Die Seiden- und Goldfäden waren gerissen, der Buchrücken war gebrochen, die Bindung defekt. Daran änderte auch die Tatsache nichts, dass der Prachtband mittlerweile bestens geschützt und in einer eigens angefertigten Archivkartonbox gelagert wird ...

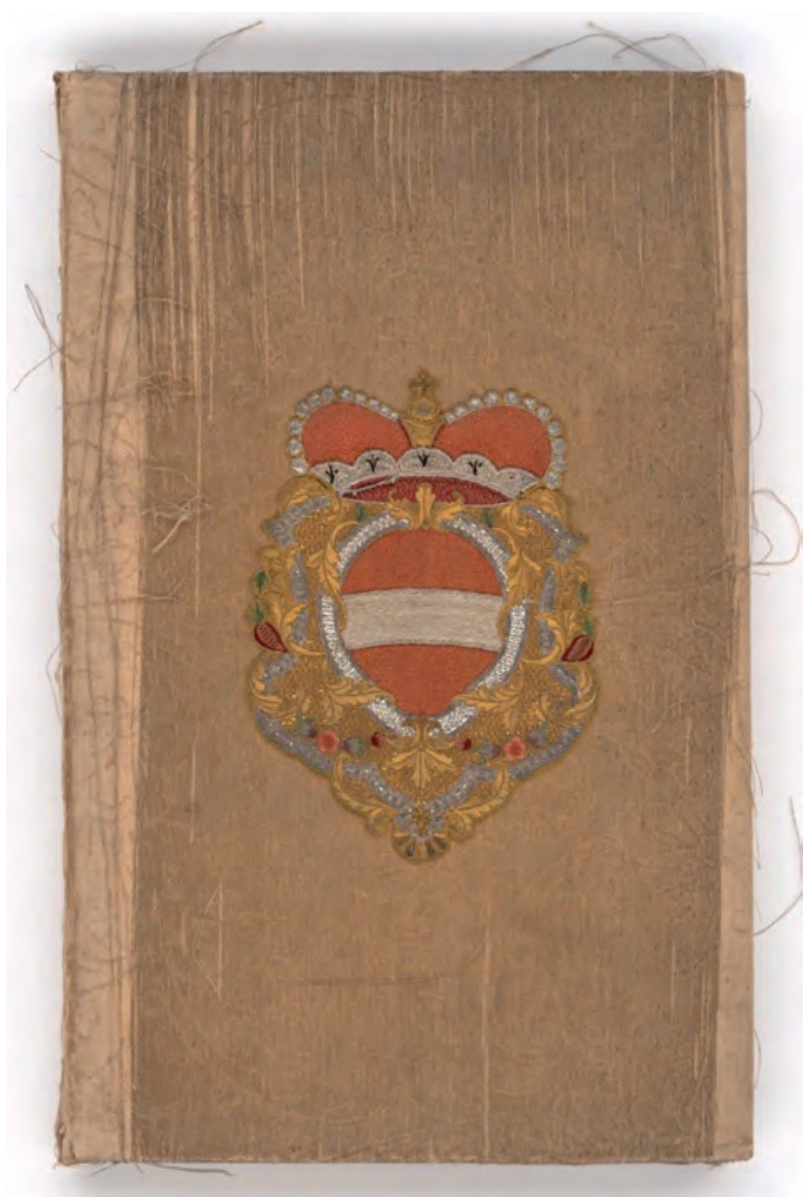

Abb. 1: Das Erbhuldigungswerk vor der Restaurierung 


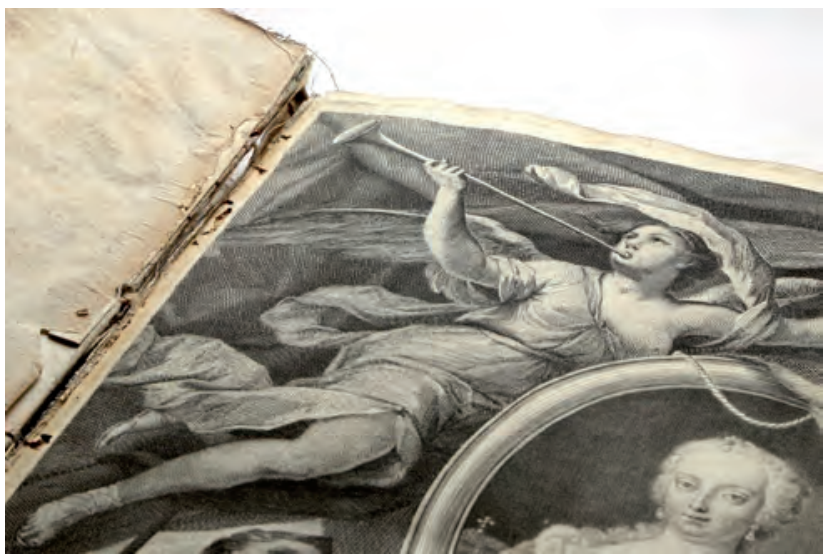

Abb. 2: Das Erbhuldigungswerk vor der Restaurierung

Eine gute Gelegenheit, dieses kostbare Werk zu restaurieren und die Restaurierung über die Crowd zu finanzieren, bot sich im Vorfeld des 300. Geburtstags von Maria Theresia am 13. Mai 2017. Wie schon 2016 zum 100. Todestag von Kaiser Franz Joseph würden zahlreiche Artikel, Reportagen, Zeitungsbeilagen, Radiosendungen und TV-Dokus zum runden Geburtstag erscheinen. Und auch die Ausstellungen zum Thema würden wieder ein Publikumsmagnet werden ... Die Kombination aus Ausstellung, Crowdfunding-Aktion ${ }^{1}$ und Medienkampagne lag also in der Luft.

Generaldirektorin Dr. Johanna Rachinger: „Die Österreichische Nationalbibliothek versteht sich als zukunftsorientiertes Informationszentrum. Neben dem großen Thema Digitalisierung wollten wir im Umfeld unserer MariaTheresia-Ausstellung auch im Bereich Sponsoring neue Wege einschlagen. Mit der Crowdfunding-Aktion sollte es Menschen ermöglicht werden, die Österreichische Nationalbibliothek bei einer ihrer wichtigsten Aufgaben zu unterstützen: der Bewahrung unseres kulturellen Gedächtnisses.“

Schon im März 2016 begannen die Vorbereitungen. Mit Unterstützung der renommierten Kommunikationsagentur Ecker \& Partner wurde in den folgenden Wochen ein umfangreicher Projektplan erarbeitet, der u.a. die Haupt- und Nebenziele festlegte, Ziel- und Dialoggruppen definierte sowie die Presse- und Werbeaktivitäten koordinierte. Mehrere Crowdfunding-Plattformen wurden sowohl hinsichtlich ihres Leistungsangebots, ihrer Referenzen und Zielgruppen, als auch ihrer Kosten verglichen. Mit www.wemakeit.com (sprich: we make it) wurde schließlich eine internationale Plattform ausgewählt, über die

1 Crowdfunding kommt von englisch crowd (Menschenmenge, Gruppe) und funding (Finanzierung), gemeint ist also die Finanzierung eines Projektes über eine möglichst große Zahl an Kleinspendern, die abhängig von der Spendenhöhe Gegenleistungen erhalten. auch schon viele deutschsprachige Kulturprojekte unterschiedlicher Institutionen und Größenordnungen erfolgreich finanziert wurden.

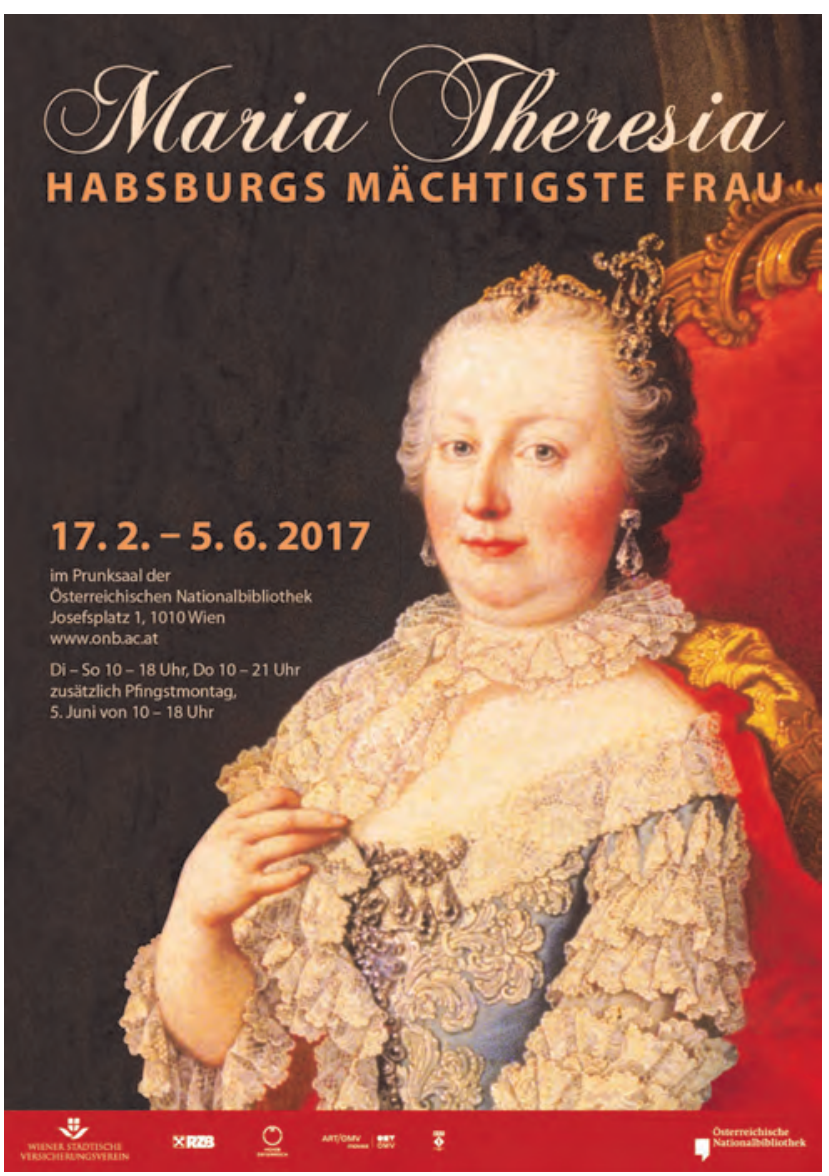

Abb. 3: In der großen Maria-Theresia-Ausstellung im Prunksaal sollte das restaurierte Werk erstmals wieder öffentlich gezeigt werden

Eine besondere Herausforderung war dabei das Konzept der Kampagne:

- Wie kann man ein möglichst breites, internetaffines und damit tendenziell jüngeres Publikum für die Erhaltung eines knapp 300 Jahre alten Buches gewinnen?

- Kann die älteste und größte Gedächtnisinstitution Österreichs potenzielle Spender mit „du“ ansprechen, wie das auf wemakeit üblich ist?

- Wie werden Stamm-Spender aus dem Kreis der „Gesellschaft der Freunde der Österreichischen Nationalbibliothek" auf eine Kampagne reagieren, zu der alle wesentlichen Informationen nur im Internet zu finden sind?

- Braucht es folglich neben Kampagnen-Video und Online-Werbung, Newsletter und Facebook auch noch das gute alte analoge Material samt Zahlschein? 


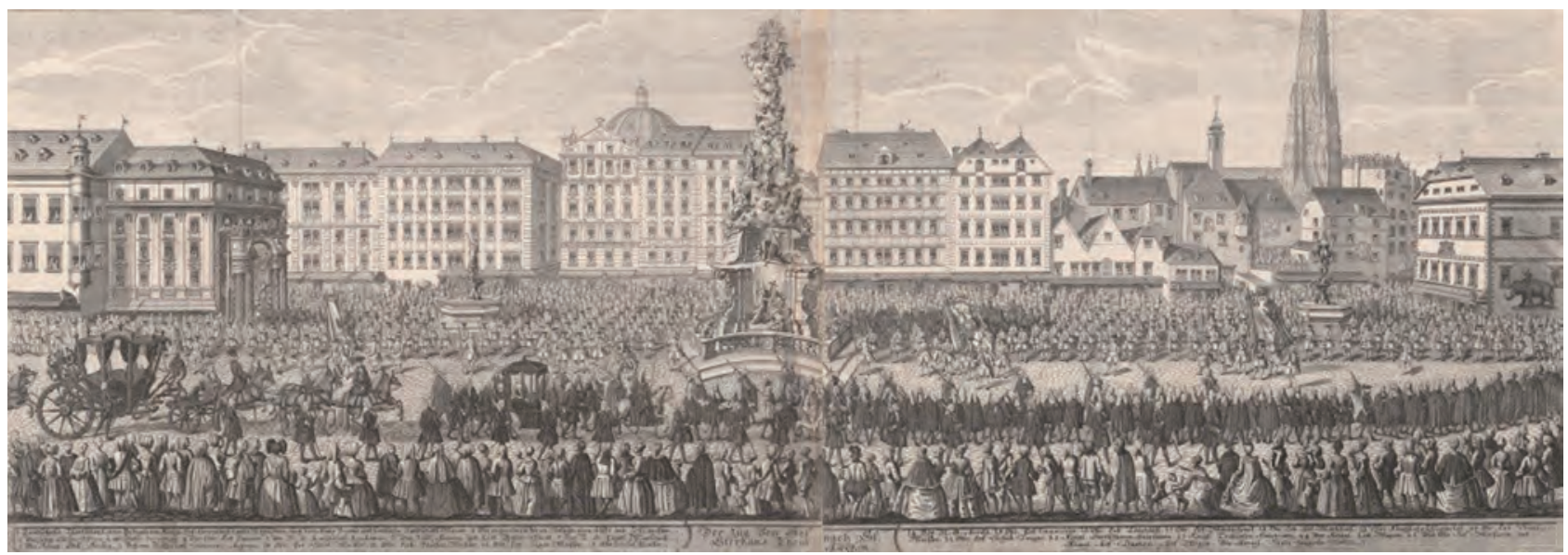

Abb. 4: Für 100 Euro Spende gab es u. a. diesen Erbhuldigungszug als Poster

- Welche „Benefits“ bietet man Spendern an, die man nicht persönlich kennt und die nicht in Wien oder Umgebung leben?

- Welche Zielsumme kann sich die Österreichische Nationalbibliothek überhaupt zutrauen, zumal einige wenige vergleichbare Kulturinstitutionen in Österreich und Europa nicht nur mit sechsstelligen, sondern auch mit fünfstelligen Zielsummen gescheitert sind $?^{2}$

Zwischen dem 20. Oktober und 22. November 2016 sollten insgesamt 15500 Euro gespendet werden. Zehn Spendenbeträge zwischen zehn und 4000 Euro waren im Angebot. Für zehn Euro erhielten die Spender etwa ein digitales Danke von Maria Theresia (ein animiertes Gif, in dem sie einem freundlich zuzwinkert). Für 100 Euro gab es einen der elf Kupferstiche aus dem Werk - den Festzug mit Maria Theresia über den Wiener Graben in Richtung Stephansdom - als exklusives Poster plus zwei Tickets für die Ausstellung und für 4000 Euro konnte man 20 Freunde oder Geschäftspartner zu einem exklusiven ChampagnerEmpfang mit Generaldirektorin Dr. Johanna Rachinger einladen - samt Preview der Ausstellung im Prunksaal und einer Führung durch die Kuratorin. ${ }^{3}$

Die 15500 Euro sollten für die Konservierung und Digitalisierung des kostbaren Werkes verwendet werden. Dank der Konservierung würde es in der Ausstellung „Maria Theresia. Habsburgs mächtigste Frau“ erstmals seit vielen Jahrzehnten wieder öffentlich gezeigt werden können und durch die Digitalisierung würde es auch un-

2 Das Ergebnis all dieser Überlegungen lässt sich auf https://wema keit.com/projects/maria-theresia-erbhuldigung abrufen.

3 Die Kampagnen-Seite auf https://wemakeit.com/projects/maria-th eresia-erbhuldigung. abhängig von der Ausstellung weltweit zur Benutzung verfügbar sein.

Schon vor Kampagnen-Start wurden drei ausgewählte Medien zu einem Hintergrundgespräch eingeladen: die Austria Presse-Agentur APA, die Tageszeitung „Der Standard" und die öffentlich-rechtliche Fernsehanstalt ORF für das Format „Wien heute“. Bei dieser Gelegenheit erklärte Generaldirektorin Dr. Johanna Rachinger die Hintergründe und Ziele der Kampagne, die Restauratorin Sabrina Bee zeigte den Journalisten das Erbhuldigungswerk im bedauernswerten Originalzustand und informierte über die Besonderheiten der Buchrestaurierung. Als Sperrfrist wurde der 20. Oktober vereinbart, der Tag, an dem die Kampagne auf wemakeit online ging, und an dem die Österreichische Nationalbibliothek auch die Presse informierte.

Kurz vor Kampagnen-Start wurden alle Mitarbeiter der Bibliothek sowie alle Guides, die in den Museen der Österreichischen Nationalbibliothek Führungen abhalten, über die Aktion informiert und eingeladen, auch selbst dafür zu werben - schließlich lebt Crowdfunding von Mundpropaganda und persönlichen Empfehlungen. „Die Crowd beginnt bei deiner Mutti" schreibt wemakeit nicht umsonst an alle Initiatoren, die auf ihrer Plattform ein Projekt starten.

Und weil der Erfolg einer Kampagne sich in den meisten Fällen im ersten Drittel der Laufzeit einstellt, wurden ab 20. Oktober alle weiteren Kommunikationskanäle bespielt: Das Video ging auf YouTube online und wurde auf Facebook gepostet. Das Pressebild war groß auf der Startseite von www.onb.ac.at platziert, ein eigener Newsletter ging an die damals über 7000 Abonnenten des monatlichen Veranstaltungsnewsletters und auf dem Online-Portal der Tageszeitung „Der Standard“ begann die Werbung zu laufen. Wichtige Stakeholder-Gruppen wie die Mitglieder der „Gesellschaft der Freunde der Österreichischen Nationalbibliothek“ bekamen zusätzlich per Post einen 
Info-Flyer, der in allen Museen des Hauses auslag und bei Veranstaltungen und Führungen verteilt wurde.

Wie bei allen großen Kampagnen kann man auch bei einer Crowdfunding-Aktion viel planen und initiieren, aber einiges ergibt sich erst im Tun und mit etwas Glück. Dazu zwei Beispiele:

Kevin Piticev, der junge Moderator des populären Radiosenders Ö3, konnte als Moderator für das Crowdfunding-Video gewonnen werden: Er, der bislang kein Naheverhältnis zur Österreichischen Nationalbibliothek hatte, fand die Aktion so spannend, dass er an seinem 24. Geburtstag eigens zum Dreh in den Prunksaal kam.

Michael Niavarani, einer der beliebtesten Kabarettisten Österreichs mit knapp 300000 Fans auf Facebook, postet dort am 26. Oktober einen Link auf wemakeit: „Zum Nationalfeiertag! Während andere glauben, sie seien Patrioten, wenn sie deutschnationale Dichter zitieren, deren Hymne im Ständestaat verwendet wurde, hab ich meinen Patriotismus anders ausgelebt: Ich habe ein Projekt der Nationalbibliothek gefunden, das ich unterstützenswert finde. Wer Lust hat kann sich das ja mal ansehen. Ich bin auf jeden Fall dabei. 2017 hat nämlich Maria Theresia Geburtstag. Habt noch einen schönen Abend, euer Nia!““ Dieser Post wurde über 50 Mal geteilt, knapp 1000 Fans haben ihn geliked und in den darauffolgenden Tagen verbreitete sich das Video wie ein Lauffeuer ...

Schon am 13. November und somit über eine Woche vor Aktionsende war das Finanzierungsziel von 15500 Euro erreicht. Bis Aktionsende gingen auf www.wemakeit. com insgesamt 18550 Euro ein. Parallel dazu erfolgten auch Spenden über Erlagscheine und Direktüberweisungen in Höhe von 7765 Euro. Insgesamt haben 166 Personen 26315 Euro gespendet. Für 112 Personen war es die erste Spende an die Österreichische Nationalbibliothek. Die meisten Spender kamen aus Wien und Niederösterreich, es nahmen aber auch Interessierte aus Tirol und Vorarlberg an der Aktion teil, elf Spenden kamen sogar aus dem Ausland.

Dank dieser großen Unterstützung konnte das Werk mittlerweile restauriert und digitalisiert werden. Der Prachtband wurde 2017 im Original in der großen MariaTheresia-Ausstellung im Prunksaal gezeigt und kann auch über den Online-Katalog „QuickSearch“ kostenlos abgerufen und genutzt werden. Die zusätzlichen Mittel flossen in die Restaurierung einer wertvollen, fast 1,5 Quadratmeter großen Druckgrafik von Johann Gottfried Haid aus dem Jahr 1760, die Maria Theresia mit ihrem Gemahl Franz Stephan und zwölf Kindern zeigt.

„Die Österreichische Nationalbibliothek hat erstmals auf Crowdfunding gesetzt, um einer neuen, jungen Zielgruppe die Möglichkeit zu bieten, schon ab zehn Euro zur

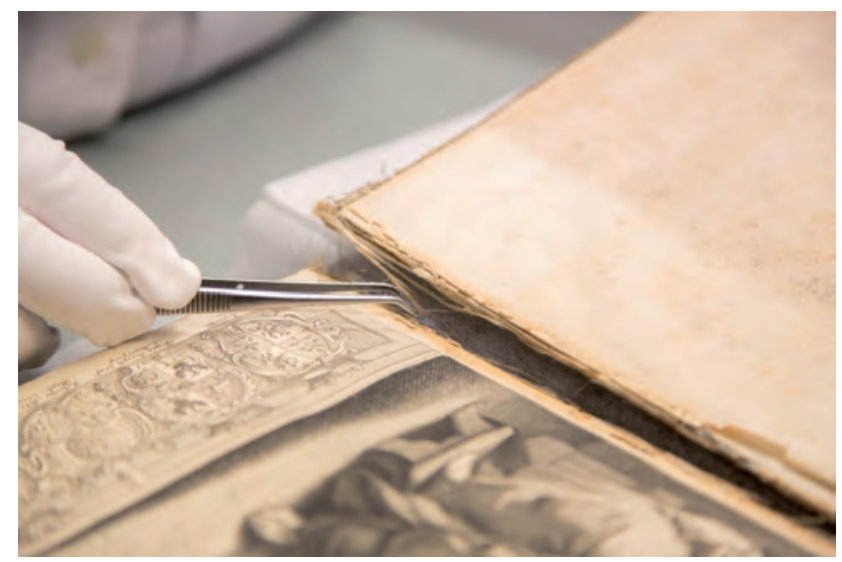

Abb. 5: In der Buchrestaurierung

Bewahrung unseres kulturellen Erbes beizutragen. Ich freue mich über diesen großen Erfolg und bedanke mich sehr herzlich bei allen Unterstützerinnen und Unterstützern für ihr Vertrauen“, so Generaldirektorin Dr. Johanna Rachinger in der abschließenden Pressemitteilung.

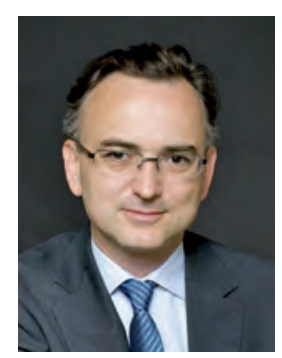

Thomas Zauner

Leiter der Abteilung Kommunikation und Marketing

Österreichische Nationalbibliothek Josefsplatz 1

A-1015 Wien

Österreich

thomas.zauner@onb.ac.at 